\title{
Molecular Cloning, Structural Characterization, and Analysis of Transcription of the Melanoma Oncogene of Xiphophorus
}

\author{
MANFRED SCHARTL AND DIETER ADAM \\ Gene Center, Max Planck Institute for Biochemistry, Am Klopferspitz 18 a, W-8033 \\ Martinsried, Federal Republic of Germany
}

Evidence for the relevance of genetic factors in melanoma formation is threefold: Firstly, there are numerous reports that melanoma occurs more frequently in certain families $(1,2,3)$ suggesting a genetic predisposition to develop the malignant disease. Secondly, specific chromosomal abnormalities have been observed accompanying early and progressing stages of melanoma $(4,5,6,7,8)$, with chromosome 1 and 6 being the most abundantly affected ones. Introduction of a normal chromosome 6 into melanoma cells led to reversion of the malignant phenotype (9). Thirdly, known oncogenes have been found to be activated in melanoma and melanoma derived cell lines, e. $\mathrm{g}$. members of the ras-gene family (10) or the src gene (11). All experimental data aimed at specifying the genetic changes that determine the neoplastic pigment cell are derived from comparisons of advanced stages of the tumor or even metastases to non-transformed melanocytes, nevi or even less well defined "normal counterparts" of the melanoma cells. The biochemical and molecular biological differences obtained through such analyses are numerous. However, it appears in general impossible to decide which of those differences are due to the primary genetic events instrumental in the causation of neoplastic transformation. Others might be important in secondary steps of tumor progression and metastases. Finally, some of the characteristics observed may be totally irrelevant for establishing and maintaining the neoplastic phenotype. They may reflect simply the chaotic molecular biology of the cancer cell. The melanoma system of Xiphophorus can be used for studies on pigment cell tumor formation with the advantage of circumventing such problems. This is because the genes that are responsible for tumor induction are clearly defined by classical genetics. In a first step towards a molecular understanding we have attempted to clone and functionally characterize the melanoma inducing oncogene.

\section{GENETICS OF MELANOMA FORMATION IN XIPHOPHORUS}

In Xiphophorus, some individuals exhibit spot patterns, composed of large, intensily black pigment cells. These cells have been termed macromelanophores while the normal sized black pigment cells that make up the uniform greyish body coloration have been designated micromelanophores (12). Already more than 60 years ago it was discovered that certain hybrids of macromelanophore pattern carrying platyfish (Xiphophorus maculatus) and of unspotted swordtail ( $X$. helleri) develop spontaneously malignant melanoma $(12,13,14)$. Shortly thereafter it was recognized that occurrence of tumors in hybrids is due to a single locus (the macromelanophore locus) of $X$. maculatus that "interacted" with the $X$. helleri genome $(15,16)$. This interaction was later on defined as the effect of modifying genes (presence of intensifying genes contributed by $X$. helleri and/or absence of repressing genes from $X$. maculatus (in the hybrid genome) which act specifically on the macromelanophore locus $(17,18,19,20,21)$.

In a typical crossing experiment (see Fig. 1) a female $X$. maculatus which carries the $X$-chromosomal macromelanophore locus Sd (Spotted dorsal, small spots in the dorsal fin) is mated to $X$. helleri, which does not carry the corresponding locus. The F1 hybrid shows enhancement of the $S d$ phenotype. Backcrossing of the $F 1$ hybrid to $X$. helleri results in offspring that segregate into $50 \%$ which have not inherited the So-locus and are phenotypically like the $X$. helleri parental strain and $50 \%$ which carry the macromelanophore locus and develop melanoma. The severity of melanoma ranges from very benign in some individuals (phenotype like the F1 hybrids) to highly malignant in others. Highly malignant melanomas of such fish grow invasive and exophytic and are fatal to the individuum. They even grow progressively following transplantation to thymusaplastic (nude) mice (22). Based on a variety of such classical crossing experiments a genetic model has been de- 

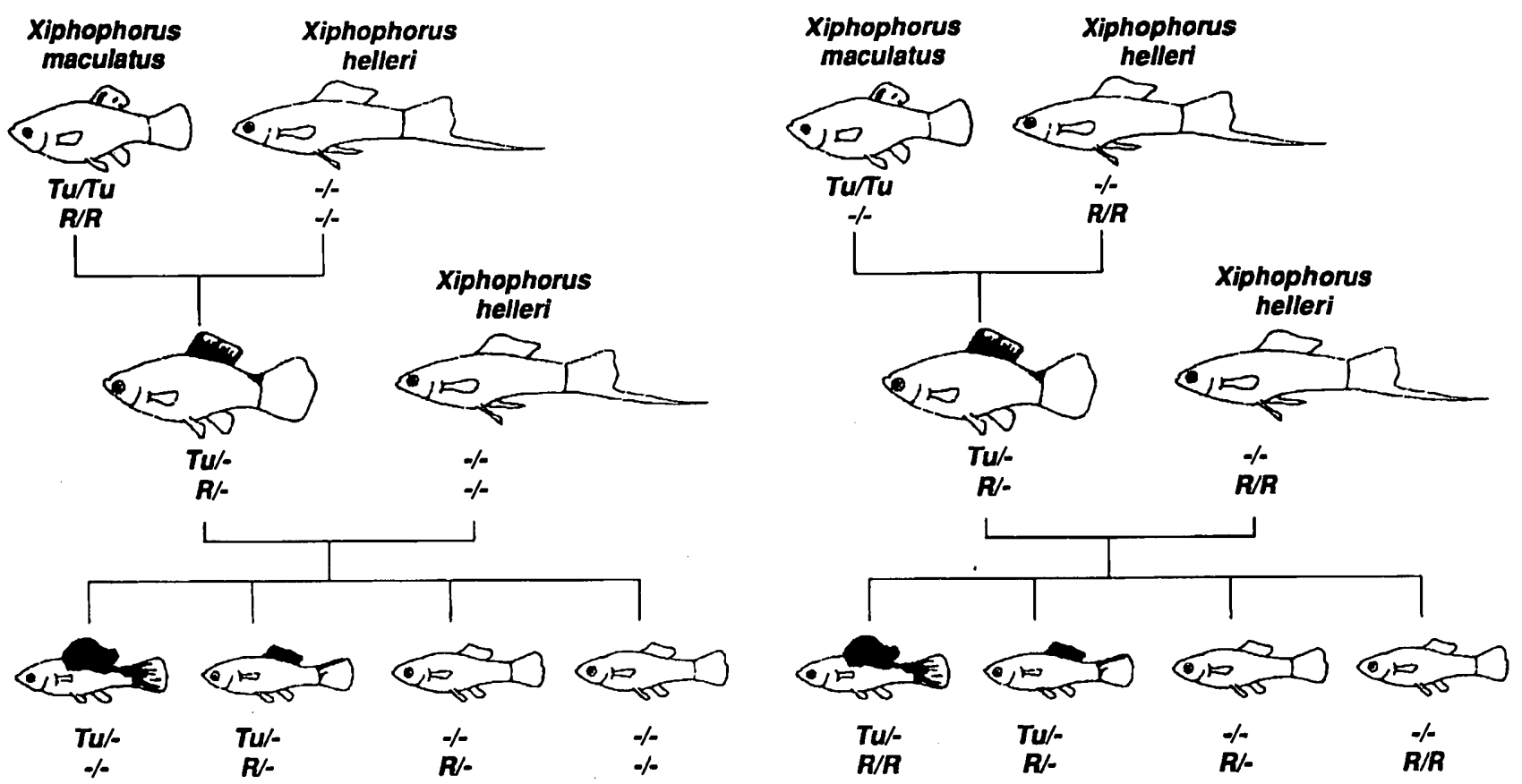

Figure 1: a) Current model to explain the classical crossing experiment leading to hybrids that develop malignant melanoma. For details see text. Tu, tumor gene, R, tumor suppressor gene. Note that the parental platyfish (Xiphophorus maculatus) carries two pigment spots in the dorsal fin that develop into melanoma in the hybrids.

b) Alternative model employing $R$ as an intensifier gene contributed by $X$. helleri in the crossing experiment.

veloped to explain tumor formation in Xiphophorus (23). The macromelanophore locus was formally equated to a sex chromosomal melanoma oncogene locus, whose critical constituent was designated "tumor-gene" (Tu). Melanoma formation then was attributed to the uncontrolled activity of Tu. In non-tumorous fish Tu activity was proposed to be negatively controlled by cellular regulatory genes or tumor suppressor genes ( $R$-genes, corresponding to the repressing modifying genes mentioned above). For the crossing experiment outlined above this means that $X$. maculatus contains the Tu-Sd locus on the X-chromosome and the corresponding major $R$ on an autosome, while $X$. helleri is proposed not to contain this particular Tu-locus and its corresponding $\boldsymbol{R}$. According to the model backcrossing of the Tu-containing hybrids to $X$. helleri results, in effect, in the progressive replacement of $R$-bearing chromosomes from $X$. maculatus by $R$ free chromosomes of $X$. helleri. The stepwise elimination of regulatory genes is thought to allow expression of the Tu phenotype, leading to benign melanoma if one functional allele of $R$ is still present or malignant melanoma if $R$ is absent (for review see 24).

It should be noted, however, that it is similarly compatible with the crossing data to attribute Tu activity to the presence of intensifying genes contributed by $X$. helleri chromosomes in the hybrid genome.

Reintroduction of suppressor genes or diluting out activating genes, respectively, by crossing malignant melanoma bearing hybrids to parental $X$. maculatus was shown to lead to a reversion of the malignant phenotype resulting in totally tumor-free fish in the succeeding backcross generations using again $X$. maculatus as the recurrent parent (24). This demonstrates that the melanoma oncogene Tu itself remains structurally unaltered during the process of activation via hybridization.

\section{REVERSE GENETIC APPROACHES TO- WARDS ISOLATION OF THE DOMINANT ME- LANOMA INDUCING GENE}

In order to understand the molecuiar basis of hereditary melanoma isolation and characterization of the genes involved was attempted. We first concentrated on Tu because in the past a large variety of different alleles from natural populations (see 25) and several spontaneous and $X$-ray induced mutants had been isolated and characterized (26). To that point no candidate gene product of TU had been characterized precluding cloning by conventional recombinant DNA technology. We therefore applied a strategy that has been termed "reverse genetics" (27) to isolate the melanoma inducing gene of $X$. maculatus. This strategy included the following steps: 1.) Determination of the chromosomal location of Tu. 2.) Identification and cloning of a molecular marker sequence for the Tu-lo- 
cus, which is apparently due to a restriction fragment length polymorphism (RFLP). 3.) Cloning of the Tucontaining region by chromosome walking or jumping. 4.) Identification and isolation of a candidate gene. 5.) Verification that the candidate gene is indeed responsible for the Tu-phenotype, namely melanoma induction in the hybrids, thereby proving that the cloned gene is actually the sought Tu gene.

After the chromosomal localization of Tu had been clearly defined by recombination and mutation analyses as to reside within the distal portion of the sex-chromosomes $(25,26)$, the most critical step was to identify a molecular marker sequence. One of several approaches (see 28) was to use heterologous oncogene/proto-oncogene probes for Southern hybridizations under conditions of low stringency. The rationale for this was that most oncogenes/proto-oncogenes of higher vertebrates fall into one of several classes of multigene families. The members of such gene families share highly conserved regions, $\theta$. g. kinase domains, DNA-binding domains etc. A molecular probe of such a conserved region detects not only all members of the gene family of the same organism under conditions of reduced stringency in Southern hybridization, but also from distantly related species, e.g. fish $(29,30,31)$. As a lot of sequences are identified in such experiments with a single probe, these are very informative with respect to the detection of RFLPs, that can be used as molecular markers. In addition, it appeared not totally illusionistic to expect that the sought dominant melanoma oncogene of Xiphophorus might be a member of one of the known oncogene/protooncogene multigene families.

From all probes tested, the viral erb B (v-erb B) probe was most informative. It is derived from the $B$ oncogene of avian erythoblastosis virus and represents an oncogenically activated version of the avian epidermal growth factor receptor (EGFR) gene (32). A probe that encompasses most of the higly conserved kinase domain, detects in EcoRI digests besides other strongly hybridizing bands two weaker bands that are only detected in the DNA of fish carrying a sex-chromosomal Tulocus, one of $6.5 \mathrm{~kb}$ cosegregating with $\mathrm{Y}$ chromosomal Tu-loci and one of $5 \mathrm{~kb}$, cosegregating with X-chromosomal Tu-loci (33). In linkage analysis employing more than 500 individual fish no recombinant between this RFLP and the Tu-locus was found $(33,34,35,36)$ indicating that this sequence is either intimately linked to Tu or even an integral part of the locus. The $5 \mathrm{~kb}$ band was cloned and found to detect besides the $\mathrm{Y}$-chromosomal $6.5 \mathrm{~kb}$ band a third hybridizing sequence of $7 \mathrm{~kb}$ which was invariably present in DNA of all fish irrespective of the presence or absence of a Tu-locus $(37,34)$ (Tab. 1). With the genomic sequence as a probe a corresponding full lenght c-DNA (35) was cloned from melanoma cells and found to encode a typical growth factor receptor protein with an extracellular ligand binding domain, a transmembrane domain and an intracellular catalytic domain, that contains all eleven structural motifs diagnostic for the protein kinase moiety of receptor tyrosine kinases (RTK). The gene was designated Xmrk for
Xiphophorus melanoma receptor kinase. It is closest related to the EGFR of higher vertebrates, but it does not represent the fish homologue of this gene (35). $\mathrm{X}$ mrk is a bona fide new member of the RTK gene family, whose physiological ligand is unknown to date.

\section{STRUCTURE AND GENOMIC ORGANIZATION OF XMRK}

In the Xiphophorus genome three different copies of the $X$ mrk gene coexist at independent genetic loci $(35,34)$. They share as far as analysed an identical exon/intron arrangement. Each locus comprises approximately $25 \mathrm{~kb}$ (see fig. 2). Xmrk also has an identical exon/intron arrangement and exon sizes as EGFR, HER $2 / n e u$ and ERB B 3 (38).

All fish, regardless of the presence or absence of the Tu-locus, contain one copy of Xmrk (recognized by the invariably present $7 \mathrm{~kb}$ EcoRl fragment, therefore called INV) on each sex-chromosome. It obviously represents a typical proto-oncogene $(35,38,34)$. The remaining two copies - named $X$ or $Y$ according to their sex-chromosomal location - are associated with macromelanophore spot pattern loci that can give rise to melanoma in the appropriate crossings (34) and are regarded as oncogenic versions of the INV copy.

All three copies of $\mathrm{X}$ mrk show a strong sequence conservation including non-coding regions (99\%). The kinase domain of the oncogenic copies does not show a single mutation which could lead to an altered protein, such mutations are restricted to the carboxy-terminus. From 122 sequence differences between the proto-oncogene and the two oncogenes found in a total of $18 \mathrm{~kb}$ of genomic sequence (exons and introns), 10 lead to amino acid exchanges, only three of which are nonconservative (38).

It is not clear at present if the observed sequence differences, or possible mutations in the so far not analysed extracellular, transmembrane and juxtamembrane domains in the $Y$ - and $X$-locus do contribute to the process of neoplastic transformation. Anyway, the mutations in the oncogenic copies of $X$ mrk are not effective as long as the genes are suppressed by the $R$-locus or not stimulated by the corresponding "activating" locus of the swordtail, respectively. Therefore such differences are not primarily responsible for bringing about the appearance of melanoma in the hybrids.

Evaluation with respect to phylogeny of the sequence differences found in all three $X$ mrk loci strongly supports the idea of a gene duplication event which created a new copy of the INV gene. This copy was translocated $2 \mathrm{cM}$ apart on the $Y$-chromosome during this duplication process and at a later stage transferred also to the $X$-chromosome by sex chromosomal crossing-over (38, see fig. 3$)$.

Verification that the additional $X$ - or Y-chromosomal copies of Xmrk are actually the critical, i.e. melanoma inducing constituent of the Tu-locus, came 
Table 1: Presence of the proto-oncogene locus (INV) and the oncogene loci $(X, Y)$ in different species and genotypes of Xiphophorus fish.

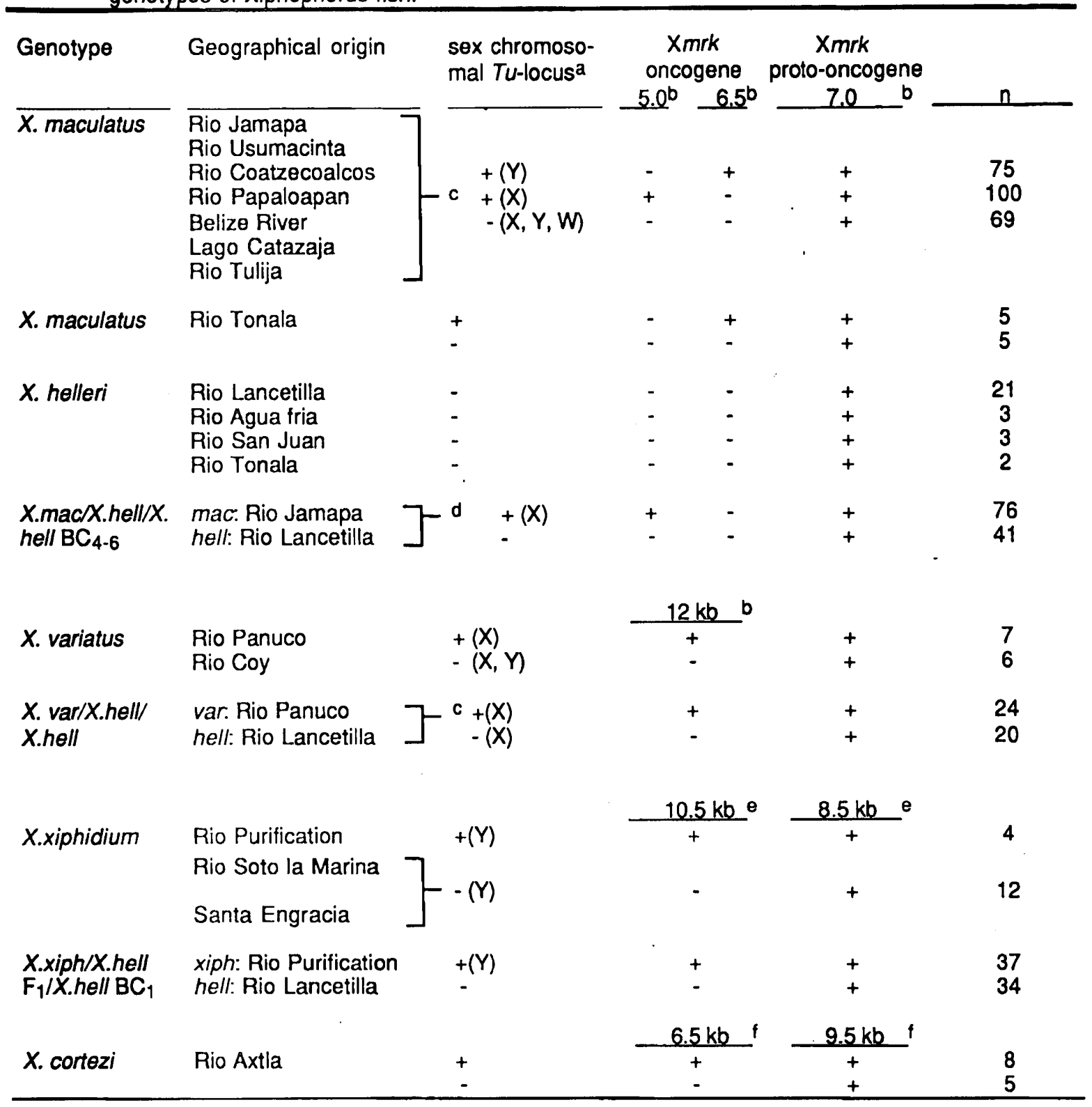

a known sex-chromosomal location in brackets

b diagnostic EcoRI restriction fragment obtained in Southern blot hybridizations with the kinase domain probes pXX21 (37) and p17-2 (35)

c for specification see ref. 34

d for details see ref. 35

e diagnostic BamHI restriction fragment obtained in Southern analyses, probes see $b$

$f$ diagnostic HindllI restriction fragment obtained in Southern analyses, probes see $b$

from analyses of "loss of function" mutants of Tu. Such mutants arise spontaneously with a very low frequency $\left(<10^{-5}\right)$ in broods of melanoma-bearing hybrids and are characterized by a loss of the ability to develop spontaneous melanoma. One such mutant was found to carry an insertion within one exon of the additional $X$-chromosomal Xmrk locus (35), resulting in the inability to develop hereditary melanoma. This showed that the Xmrk oncogene is necessary for tumorigenesis and therefore is the critical constituent of the Tu-locus. 


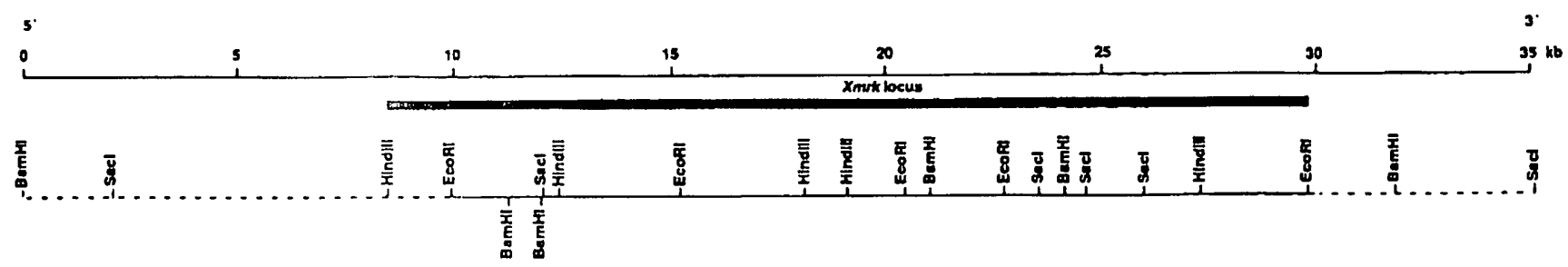

Figure 2: Restriction map of the Xmrk locus (shown by example of the INV locus). Cloned regions are indicated by solid lines, the gray bar indicates the promoter region, the black bar marks the transcribed part of the locus.

\section{TRANSCRIPTIONAL ACTIVATION OF THE XMRK ONCOGENE}

The Xmrk genes give rise to two transcripts of different size, one of $5.8 \mathrm{~kb}$ and one of $4.7 \mathrm{~kb}$. The shorter transcripts are product of the oncogenic $X$ - and $Y$-chromosomal Xmrk copies while the $5.8 \mathrm{~kb}$ mRNA is transcribed from the proto-oncogenic INV copy $(35,38)$.

The proto-oncogene transcript is highly abundant as maternal RNA in unfertilized eggs and is differentially expressed during organogenesis. In adult nontumorous fish, expression of the INV gene is restricted to low levels of transcripts in skin, fins and gills. Low levels of the $5.8 \mathrm{~kb}$ transcript are also found in melanoma (38). This expression is not influenced by presence or absence of the $R$-locus, as the $5.8 \mathrm{~kb}$ transcript is found at similar levels in melanomas of differing malignancy.

In contrast, expression of the $X$ - and $Y$-copy of $X$ mrk is depending on the absence or presence of the $A$-locus. Expression of the $4.7 \mathrm{~kb}$ transcript is limited to melanoma, it cannot be detected in any other tissue. The degree of Xmrk oncogene expression and the malignancy of melanoma are definitely correlated: the amount of the $4.7 \mathrm{~kb}$ transcript is low in benign and very high in malignant melanomas $(35,38)$. Overexpression of the oncogenic Xmrk copies seems to be a prerequisite for tumor formation.

Analysis of the genomic sequences to explain the size difference of $1.1 \mathrm{~kb}$ of the INV transcript on the one, and the $X$ - and $Y$-transcript on the other side, revealed intruiging differences in the 5 'region. Both oncogenic copies of Xmrk use a transcription start site located closer to the ATG codon than that used by the proto-oncogene. This is due to the presence of two different promoters in the different Xmrk genes (38). The promoter of the oncogenic Xmrk loci is obviously only active in the melanoma cells of the hybrid fish but appears inactive in the purebred parental fish.

Based on this results, it is conceivable to argue that the R-locus is somehow involved in transcriptional control of the promoter of the oncogenic Xmrk loci (X, $Y)$. Loss of the R-locus from the platyfish or gain of its swordtail counterpart, respectively, then would lead to uncontrolled expression of $X$ and $Y$ resulting in melanoma formation.

\section{IMPLICATIONS AND PERSPECTIVES}

The classical model (23) to explain spontaneous melanoma formation in platyfish/swordtail hybrids employing the sex-chromosomal dominant acting oncogene locus $T u$, which we have shown to encode a copy of the $X$ mrk gene, and the autosomal tumor suppressor locus $R$, has been extended to explain also a variety of other phenomena and experimental observations such as the formation of carcinogen-and $X$-ray-induced tumors of all histiotypes in Xiphophorus hybrids, and the occurence of macromelanophore spot pattern in several other Xiphophorus species besides $X$. maculatus, some of which predispose to spontaneous melanoma formation in hybrids while others do not (39). After cloning of the Xmrk gene from the Tu-locus these problems can now be approached experimentally.

The extended model to explain induction of tumors of all etiologies, which was even stretched to a unified concept for the origins of cancers in all multicellular organisms ranging from plants to man (39), was faced with the problem that carcinogen treatment led to tumor induction also in hybrids that did not contain a sex-chromosomal Tu-locus associated with the macromelanophore locus. It was therefore proposed that $T u$ is present in the genome of Xiphophorus in multiple copies, spread over all chromosomes. The macromelanophore locus associated Tu-copy was termed "accessory Tu", because it is obviously lacking in a variety of genotypes, e. g. the unspotted swordtails, without any negative effect, while the autosomal copies were termed "indispensablen". These were pro-

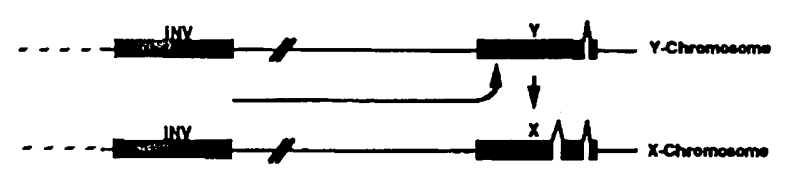

Figure 3: Model for the generation of the oncogenic $X$ mrk copies $(X, Y)$ by gene duplication of the proto-oncogenic INV Xmrk gene and sex chromosomal crossing-over (indicated by arrows). Large deletions are indicated by gaps. 
posed to encode the information for neoplastic transformation realized in most of the carcinogen-induced tumors in addition to their so far undefined important physiological function. Because the indispensable copies of $T u$ were not easily recognizable by macromelanophore patterns, their existance remained hypothetical. The genomic organization of Xmrk clearly is not in accordance with these considerations on "accessory" and "indispensable" Tu copies. Xmrk is only present on the sex-chromosomes (34). If activated oncogenes responsible for tumor induction after carcinogen treatment could not be mapped to the sexchromosome (40), they are definitely distinct from Xmrk and are consequently not encoded by Tu. Further evidence for this issue may be obtained from studies on $X$ mrk expression in tumors of different etiology and also from molecular analysis of the factors responsible for tumor formation following carcinogen treatment.

The idea that one oncogene, namely Tu, should be responsible for a large variety of tumors of different histogenesis, is also not supported by findings in other systems. Melanoma in transgenic mice were obtained due to the activity of the SV40 T-antigen, which is clearly not responsible for most other melanoma (41). Different oncogenes have been found activated in tumors even of identical histiotype $(10,42)$.

With respect to the phenotypic diversity of macromelanophore patterns in the feral Xiphophorus populations it was reasoned that $T u$ itself specifies the phenotype of the macromelanophore. Pattern information was proposed to be encoded in a series of closely linked "compartment" genes. The potential for melanoma induction was thought to depend on the chromosomal location of the major $R$-gene being closely linked to the Tu-locus in those cases, where a macromelanophore locus does not have a malignant potential. In the case of melanoma predisposing loci $A$ was proposed to be located on an autosome, thus being separated from $T u$ in backcross hybrids, analogous to the situation described for "Spotted dorsal" of the Rio Jamapa platyfish. Again studies on the genomic organization of Xmrk led to a different view (34).

The genes determining the phenotype of the macromelanophore are different from Xmrk although closely linked. In a total of 13 sex-chromosomal macromelanophore loci, that give rise to enhanced pigmentation and melanoma following the appropriate crossings, always an additonal copy of Xmrk was found (Table 1). Those macromelanophore loci, which do not predispose for melanoma formation simply do not contain the additional, oncogenic copy of Xmrk. In fish with those loci only the proto-oncogene INV of $X m r k$ is present (34). In the melanoma predisposing macromelanophore loci the linkage of the oncogenic $X m r k$ copy to the pigmentation gene(s) is so tight, that it appears possible to identify these genes by chromosome walking or jumping using $X m r k$ as a starting point.

The identity of the Xmrk gene product as a putative novel growth factor receptor with a tyrosine kinase activity implicates further questions the answers to which should help to understand how overexpression of the gene mediates the initiation and maintenance of the neoplastic phenotype of pigment cells. It will be important to find those cellular substrates for the Xmrk kinase that transduce the mitogenic signal exerted by the Xmrk ligand to the nucleus as well as to identify the genes that are activated following Xmrk stimulation. Isolation and characterization of the ligand will not only help to elucidate the normal, physiological function of $\mathrm{Xmrk}$ but also help to investigate if the melanoma cells constitute an autocrine growth stimulatory loop sensu Todaro and/or if the oncogenic Xmrk loci encode an "activated" mutant protein, that is constitutively active.

So far, it was not possible to decide if Tu activity is suppressed by $R$-alleles from platyfish or enhanced by corresponding alleles of the swordtail. Analysis of the promoter of the oncogenic Xmrk copies will reveal "enhancer" or "silencer" elements, that are involved in the transcriptional control of the gene in the melanoma cells. This will help to decide which model (see Fig. 1) is correct.

To obtain information how the oncogenic activity of the $X$-and $Y$-copies of $X m r k$ is suppressed in the parental purebred fish or, alternatively, is enhanced in the hybrids, isolation and characterization of the $R$ encoded gene(s) is required. The finding that transcriptional control may be the mechanism through which $R$ regulates $X$ mrk emphasises the importance of identifying the factors that control transcription of the oncogenic Xmrk copies. An alternative approach will be to use also for cloning of the $R$ encoded gene(s) the methodology of reverse genetics.

Melanomas in feral Xiphophorus populations are extremely rare $(43,44)$, however, their existence gives additional significance to the reasoning that the duplicated Xmrk genes that reside closely to the macromelanophore locus, are potentially injurious. It will be important to exploit also from an evolutionary and social behavioural genetic point of view how such a potential deleterious gene has been maintained in the natural populations of most species of Xiphophorus.

\section{ACKNOWLEDGEMENTS}

We thank A. Geishauser for breeding of the fish, and S. Romanow for typing the manuscript. This work was supported by the Bundesministerium für Forschung und Technologie through Schwerpunkt "Grundlagen und Anwendungen der Gentechnologie" (Grant No 26), the Deutsche Forschungsgemeinschaft through Schwerpunkt "Molekulare Tumorzytogenetik" (Scha 408/2-3) and SFB 190, the Stiftung Volkswagenwerk "Wettbewerb Biowissenschaften", the Max-PlanckGesellschaft, and a "Heisenberg" fellowship to M. S. 


\section{Reforences:}

1. Lynch HT, Fusaro RM, Pester J, Lynch, JF. Familial atypical multiple mole melanoma (FAMMM) syndrome: Genetic heterogeneity and malignant melanoma. Br. J. Cancer 1980:42:58-70.

2. Traupe H, Macher E. Classical and molecular genetics of malignant melanoma and dysplastic nevi. In: Elwood JM, ed. Melanoma and nevi - incidence, interrelationships and implications. Basel: Karger, 1988: 77-94.

3. Rhodes AR, Harrist TJ, Day CL, et al. Dysplastic melanocytic nevi in histologic association with 234 primary cutaneous melanomas. J. Am. Acad. Dermatol. 1983:9:563-74

4. Trent JM, Rosenfeld SB, Meyskens FL. Chromosome $6 q$ involvement in human malignant melanoma. Cancer Genet. Gytogenet. 1983:9:17780.

5. Balaban GB, Herlyn M, Clark WH, Nowell PC. Karyotypic evolution in human malignant melanoma. Cancer Genet. Cytogenet. 1986:19:11322.

6. Pedersen MI, Bennett JW, Wang N. Nonrandom chromosome structural aberrations and oncogene loci in human malignant melanoma. Cancer Genet. Cytogenet. 1986:20:11-27.

7. Limon J, Dal Cin P, Sait SNJ, Karakousis C, Sandberg AA. Chromosome changes in metastatic human melanoma. Cancer Genet. Cytogenet. 1988:30:201-11.

8. Dracopoli NC, Alhadeff B, Houghton AN, Old LJ. Loss of heterozygosity at autosomal and $X$-linked loci during tumor progression in a patient with melanoma. Cancer Research 1987:47:3995-4000.

9. Trent JM, Stanbridge EJ, McBride HL, et al. Tumorigenicity in human melanoma cell lines controlled by introduction of human chromosome 6. Science 1990:247: 568-71.

10. Albino AP, Nanus DM, Mentle IR, et al. Analysis of ras oncogenes in malignant melanoma and precursor lesions: correlation of point mutations with differentiation phenotype. Oncogene 1989:4: 1363-74.

11. Barnekow A, Paul E, Schartl M. Expression of the c-src Protooncogene in Human Skin Tumors. Cancer Research 1987:47: 235-40.

12. Gordon $M$. The genetics of a viviparous top-minnow Platypoecilus: The inheritance of two kinds of melanophores. Genetics 1927:12:253-83.

13. Hãussler $G$. Über Melanombildungen bei Bastarden von Xiphophorus maculatus var. rubra. Klin. Wochenschr. 1928:7:1561-62.

14. Kosswig $\mathrm{C}$. Über Kreuzungen zwischen den Teleostiern Xiphophorus helleri und Platypoecilus maculatus. $Z$. Indukt. Abstammungs.-Vererbungsl. 1928:47:150-8.

15. Gordon $M$. Hereditary basis of melanosis in hybrid fishes. Am. J. Cancer 1931:15:1495-1519.

16. Kosswig C. Das Gen in fremder Erbmasse. Züchter 1929:1:152-157.

17. Gordon M. A genetic concept for the origin of melanomas. Ann. N.Y. Acad. Sci. 1958:71:1213-22.

18. Atz JW. Effects of hybridization on pigmentation in fishes of the genus Xiphophorus. Zoologica 1962:47:153-81.

19. Kosswig C. Genetische Grundlagen des Polymorphismus. Zool. Anz. 1965:175:21-50.

20. Zander CD. Über die Entstehung und Veränderung von Farbmustern in der Gattung Xiphophorus (Pisces). Mitt. Hamburg Zool. Mus. Inst. 1969:66:241-71.

21. Kallman KD. Different genetic basis of identical pigment patterns in two populations of platyfish, Xiphophorus maculatus. Copeia 1970:3:472-87.

22. Schartl M, Peter RU. Progressive growth of fish tumors after transplantation into thymus-aplastic (nu/nu) mice. Cancer Res. 1988:48:741-44.

23. Ahuja MR, Anders F. A genetic concept of the origin of cancer, based in part upon studies of neoplasms in fishes. Prog. Exp. Tumor Res. 1976:20: 380-97.

24. Anders F, Schart M, Barnekow A, Anders A. Xiphophorus as an in vivo model for studies on normal and defective control of oncogenes. Adv. Cancer Res. 1984:42:191-275.

25. Kallman KD. The platyfish, Xiphophorus maculatus. In: King RC, ed. Handbook of Genetics: 4. New York: Plenum Press, 1975:8-132.

26. Anders A, Anders F, Klinke K. Regulation of gene expression in the Gordon-Kosswig melanoma system I, II. In: Schröder JH, ed. Genetics and Mutagenesis of Fish. New York: Springer-Verlag, 1973: 33-63.

27. Orkin SJ. Reverse genetics and human disease. Cell 1986:47:845-50.

28. Schartl M, Wittbrodt J, Mäueler W, et al. Oncogenes and melanoma formation in Xiphophorus (Teleostei: Poeciliidae). In: Schröder $\mathrm{JH}$, Schartl M, eds. New Trends in Ichthyology. Hamburg: Parey, 1991: in press.

29. Mäueler W, Raulf F, Schartl $M$. Expression of proto-oncogenes in embryonic, adult, and transformed tissue of Xiphophorus (Teleostei: Poeciliidae). Oncogene 1988:2:421-30.

30. Mäueler W, Barnekow A, Eigenbrodt $E$, et al: Different regulation of oncogene expression in tumor and embryonal cells of Xiphophorus. Oncogene 1988:3:113-22.

31. Hannig G, Ottilie S, SchartI M. Conservation of structure and expression of the c-yes and fyn genes in lower vertebrates. Oncogene 1991:in press.

32. Ullrich A, Coussens L, Hayflick JS, ot al. Human epidermal growth factor receptor cDNA sequence and aberrant expression of the amplified gene in A431 epidermoid carcinoma cells. Nature 1984:309:418-25.

33. Schartl M. A sex chromosomal restriction-fragment-length marker linked to melanoma-determining $\mathrm{Tu}$ loci in Xiphophorus. Genetics 1988:119:679-85.

34. Schartl M. Homology of melanoma-inducing loci in the genus Xiphophorus. Genetics 1990:126:108391.

35. Wittbrodt J, Adam D, Malitschek B, et al. Novel putative receptor tyrosine kinase encoded by the melanoma-inducing Tu locus in Xiphophorus. Nature 1989:341:415-21. 
36. Zechel C, Schleenbecker U, Anders A, Anders F. $v$-erbB related sequences in Xiphophorus that map to melanoma determining Mendelian loci and overexpress in a melanoma cell line. Oncogene 1988:3:605-17.

37. Adam D, Wittbrodt J, Telling A, Schartl M. RFLP for an EGF-receptor related gene associated with the melanoma oncogene locus of Xiphophorus maculatus. Nucl. Acids Res. 1988:16:7212.

38. Adam D, Mäueler W, Schartl M. Transcriptional activation of the melanoma inducing Xmrk oncogene in Xiphophorus. Oncogene 1991:6:73-80.

39. Anders F. A biologist's view of human cancer. In: Neth $R$, et al., eds. Modern Trends in Human Leukernia 8. Berlin: Springer Verlag, 1990: XXIIIXIV.

40. Schwab M, Abdo S, Ahuja MR, et al. Genetics of susceptibility in the platyfish/swordtail tumor system to develop fibrosarcoma and rhabdomyosarcoma following treatment with $\mathrm{N}$-methyl-N-nitrosourea (MNU). Z. Krebsiorsch. 1978:91:301-15

41. Bradl M, Klein-Szanto A, Porter S, Mintz B. Malignant melanoma in transgenic mice. Proc. Natl. Acad. Sci. USA 1991:88:164-8.

42. Slamon DJ, deKernion JB, Verma IM, Cline $M$. Expression of cellular oncogenes in human malignancies. Science 1984:224:256-62

43. Borowsky RL. Melanomas in Xiphophorus variatus (Pisces, Poeciliidae) in the absence of hybridization. Experientia 1973:29:1431-3.

44. Kallman KD. Inheritance of melanophore patterns and sex determination in the montezuma swordtail, Xiphophorus montezumae cortezi Rosen. Zoologica (N.Y.) 1971:56:77-94. 